\title{
Localization transition on complex networks via spectral statistics
}

\author{
M. Sade, T. Kalisky, S. Havlin and R. Berkovits \\ The Minerva Center, Department of Physics, Bar-Ilan University, Ramat-Gan 52900, Israel
}

(Dated: Oct. 21, 2005, version 3.1)

\begin{abstract}
The spectral statistics of complex networks are numerically studied. The features of the Anderson metal-insulator transition are found to be similar for a wide range of different networks. A metalinsulator transition as a function of the disorder can be observed for different classes of complex networks for which the average connectivity is small. The critical index of the transition corresponds to the mean field expectation. When the connectivity is higher, the amount of disorder needed to reach a certain degree of localization is proportional to the average connectivity, though a precise transition cannot be identified. The absence of a clear transition at high connectivity is probably due to the very compact structure of the highly connected networks, resulting in a small diameter even for a large number of sites.
\end{abstract}

PACS numbers: $89.75 . \mathrm{Hc}, 72.15 . \mathrm{Rn}$

The Anderson transition predicts a transition from extended (metallic) to localized (insulating) eigenstates as a function of the disorder or energy of a quantum system [1]. This second-order phase transition turns out to be a very general property related to the transport of quantum and classical waves in disordered systems and signatures of it have been observed for electrons in metals, microwaves in waveguides, light in liquids and gels and acoustical waves in the earth crust [2]. For all these systems the clearest signature of the transition is the different spread of a signal injected into the system. While in a metallic phase the injected wave will spread all over the system, in the insulating phase it will be localized in the vicinity of the injection point. This effect is a result of the constructive interference between time-reversed path throughout the system. Thus, the details of the transition are strongly influenced by the dimensionality and topology of the system.

The lower critical dimension, below which the system is localized for all values of disorder, is believed to be two [3] , since the probability of returning to the origin (i.e., constructive interference due to time reversal symmetry) is finite below $d=2$. The upper critical dimension [4] (beyond which the critical exponents reach their meanfield values) remains uncertain although it is argued to be infinity [5]. The parameters defining the transition are traditionally given as the critical disorder $W_{c}$ expressed in terms of the width of the distribution from which the on-sites energies in the Anderson model are drawn, and the critical exponent $\nu$ (for definitions see Sec. II). For square lattices with dimensionality $d=3,4$ the values of $W_{c}$ and $\nu$ are well established : for $d=3, W_{c} \sim 16.5$ and $\nu \sim 1.5$ [6, 7], while for $d=4, W_{c} \sim 35$ and $\nu \sim 1$ [8, 9]. For higher dimensions the following extrapolation was offered [9]: $\nu \sim 0.8 /(d-2)+0.5$ and $W_{c} \sim 16.5(d-2)$, which was obtained by studying the transition on bifractal topologies. Thus, the mean field critical index value of $1 / 2$ is obtained in the the upper critical dimension $d=\infty$.

There has been recently much interest in the properties of random scale-free networks 10, 11, 12, 13, 14,
15, 16, 17, 18, 19. These networks are characterized by the fact that each node is connected with some finite probability to any other node in the graph, which is very different from the usual topology of a real space lattice in which nodes are connected only to their neighbors. This leads to a very interesting behavior of the graphs when properties such as percolation, cluster structures, paths length etc. are considered [11]. Interest in the influence this unusual topology has on the properties of wave interference (i.e., the Anderson transition of these graphs) is rising. Indeed, recently the Anderson transition in particular networks, namely the small-world networks [20, 21] and the Cayley tree 22] were studied. Older work on the localization properties of sparse random matrices [23] is directly relevant to Erdös-Rényi graphs. Since scale free networks have an unusual topology for which anomalous classical properties have been found 24, 25] it is of particular interest to study the Anderson localization in these networks.

Essentially, the probability to return to the origin defines the dimensionality of a system for the Anderson transition. Therefore, one may speculate that random graphs, which have only very long closed trajectories, correspond to systems with an infinite dimension [26]. On the other hand, the critical disorder which depends roughly on the number of nearest neighbors $Z$ is expected to follow [1] $W_{c} \sim Z$, which for a random graph with an average degree $\langle k\rangle$ (i.e., the average number of connections per node) corresponds to $W_{c} \sim\langle k\rangle$. Thus, one may expect here an interesting situation in which the critical index $\nu$, which is determined by the dimensionality is close to a half, while the critical disorder is determined by $\langle k\rangle$. This is very different than the situation described by the extrapolation given above, where the critical disorder for an infinite dimension should also be infinite.

Beyond the general interest in investigating the Anderson transition on scale free networks, and the fresh outlook it might provide on the localization phenomenon, the metal-insulator transition can provide insights into the functionality of complex networks. Consider for example an optical communication network. In such a net- 
work the edges of the graph represent optical fibers in which light propagates and the nodes represents a beam splitter which redistributes the incoming wave into the outgoing bonds connected to the node. Since for high quality optical fiber there are essentially no losses or decoherence on the bonds, the amplitude of the transmitted wave will not depend on the bond length (on the other hand phase will depend on the length). This network may be mapped on a tight binding Hamiltonian of the type described in Sec. [II 27]. An interesting question for such a scale free network is whether a wave injected into one of the nodes will produce a signal at all other node (which in the language of the Anderson localization is equivalent to the question is the system metallic) or only at a finite set of other nodes (i.e., the system is insulating). A metal-insulator transition in this network corresponds to a phase transition between those two phases as function of the properties of the nodes. Generally, for any complex network in which information propagates in a wave-like fashion and interference is possible, the Anderson transition will limit the spread of the information throughout the network.

The paper is organized in the following way: In the next section (Sec. I) we describe the different networks which were considered, while in Sec II the spectral statistics method which was applied in order to identify the metal-insulator transition is outlined. In Sec. III the results are depicted and some general characteristics of the localization on complex networks are discussed.

\section{CHARACTERISTICS OF THE DIFFERENT NETWORKS}

Our main goal is to study the Anderson transition for different complex networks. In this section we shall define the characteristics of the networks which will be considered.

\section{A. Random Graph}

A random graph (or- random regular graph) is a graph with $N$ nodes, each is connected to exactly $k$ random neighbors [11]. The diameter of a graph is the maximal distance between any pair of its nodes. In a random graph the diameter $d$ is proportional to $\ln N$. In Sec. III we shall present results of the level spacing distribution for random-regular graphs with $k=3$.

\section{B. Erdös-Rényi Graphs}

In their classical model from 1959 Erdös and Rényi (ER) 28] describe a graph with $N$ nodes where every pair of nodes is connected with probability $p$ resulting in $\langle k\rangle=$ $N p$. For a large random graph the degree distribution follows the Poisson distribution:

$$
P(k)=e^{-\langle k\rangle} \frac{\langle k\rangle^{k}}{k !} .
$$

The diameter of such a graph follows: $d \sim \ln N$, similar to a random graph. In Sec. III we have specifically calculated the level distribution for $\langle k\rangle=$ $3,3.1,3.2,3.5,4,5,7.5$ and 10 .

\section{Scale-Free Networks}

Scale free (SF) networks 10 are networks where the degree distribution (i.e., fraction of sites with $\mathrm{k}$ connections) decays as a power-law. The degree distribution is given by 29] :

$$
P(k)=c k^{-\lambda}, m<k<K
$$

where $c=(\lambda-1) m^{\lambda-1}$ and $K=m N^{1 /(\lambda-1)}$ [14], $\lambda$ is the power-law exponent, $m$ is a lower cutoff, and $K$ is an upper cutoff. Thus, there are no sites with degree below $m$ or above $K$. The diameter of the SF network can be regarded as the mean distance of the sites from the site with the highest degree. For graphs with $2<$ $\lambda<3$ the distance behaves as $d \sim \ln (\ln (N))$ [15], and for $\lambda=3$ as $d \sim \ln (N) / \ln (\ln (N))$ 16. This anomalous behavior stems from the structure of the network where a small core containing most of the high degree sites has a very small diameter. For higher values of $\lambda$ the distance behaves as in ER, i.e., $d \sim \ln (N)$. The $\langle k\rangle$ of a SF graph is obtained by the following expression:

$$
\langle k\rangle=\frac{1-\lambda}{2-\lambda} \times \frac{K^{2-\lambda}-m^{2-\lambda}}{K^{1-\lambda}-m^{1-\lambda}}
$$

For $\lambda>2$ and large enough $N$ the average degree , $\langle k\rangle$, is a constant. The SF networks analyzed in this paper correspond to $\lambda=3.5,4,5$ with $m=2$ (lower cutoff), and $\lambda=4,5$ with $m=3$. Due to their small diameter, SF networks with $\lambda<3$ were not considered.

\section{Double peaked distributions}

In order to find hierarchical relation between the different graphs, we studied also some variations on these graphs. For a random graph we changed the degree of a small percent of the nodes, so we have a graph with double peaked distribution. Thus, the average connectivity, $\langle k\rangle$, is the average degree of the nodes. Several examples were taken: changing $5 \%$ of the nodes to $k=5$ (instead of $k=3$ ) resulting in $\langle k\rangle=3.1$, or changing $5 \%$ of the nodes to $k=10(\langle k\rangle=3.35)$. Replacing $20 \%$ of the nodes connectivity for the previous cases will result in $\langle k\rangle=3.4$ (for $k=5$ nodes) and $\langle k\rangle=4.4$ (for $k=10$ nodes). Additionally, in order to relate with previous results of the metal insulator transition on a Cayley-tree 
22 we checked a tree in which $5 \%$ of its nodes have higher degree $(k=4)$ resulting in an average connectivity 3.05 and creating few closed trajectories - loops.

\section{METHOD}

Now we turn to the calculation of the spectral statistics of these networks. First, one must construct the appropriate network structure, i.e., to determine which node is connected to which. This is achieved using the following algorithm [14, 17]:

1. For each site choose a degree from the required distribution.

2. Create a list in which each site is repeated as many times as its degree.

3. Choose randomly two sites from the list and connect this pair of site as long as they are different sites.

4. Remove the pair from the list. Return to 3.

The diameter of a graph is calculated by building shells of sites [29]. The inner shell contains the node with the highest degree, the next contains all of its neighbors, and so on. Of course, each node is counted only once. The diameter of the system is then determined by the number of shells. Two more options which were considered are defining the diameter by the most highly populated shell, or by averaging over the shells. The diameter obtained by the various methods are quite similar.

The energy spectrum is calculated using the usual tight-binding Hamiltonian,

$$
H=\sum_{i} \varepsilon_{i} a_{i}^{\dagger} a_{i}-\sum_{\langle i, j\rangle} a_{j}^{\dagger} a_{i},
$$

where first term of the Hamiltonian stands for the disordered on-site potential on each node $i$ of the network. The on-site energies, $\varepsilon_{i}$ are uniformly distributed over the range $-W / 2 \leq \varepsilon_{i} \leq W / 2$. The second term corresponds to the hopping matrix element which is set to 1 , and $\langle i, j\rangle$ denotes nearest neighbor nodes which are determined according to the network structure. We diagonalize the Hamiltonian exactly, and obtain $N$ eigenvalues $E_{i}$ (where $N$ is the number of nodes in the graph) and eigenvectors $\psi_{i}$. Then we calculate the distribution $P(s)$ of adjacent level spacings $s$, where $s=\left(E_{i+1}-E_{i}\right) /\left\langle E_{i+1}-E_{i}\right\rangle$, and $\langle\ldots\rangle$ denotes averaging over different realizations of disorder and when relevant also over different realizations of node connectivities.

One expects the distribution to shift as function of the on-site disorder from the Wigner surmise distribution (characteristic of extended states):

$$
P_{W}(s)=\frac{\pi s}{2} \exp \left[-\frac{\pi s^{2}}{4}\right],
$$

at weak disorder to a Poisson distribution (characteristic of localized states) at strong disorder:

$$
P_{P}(s)=e^{-s} .
$$

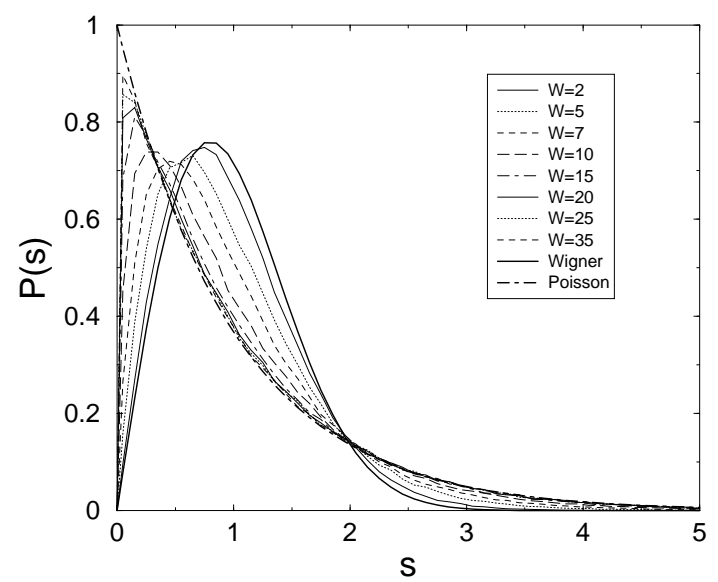

FIG. 1: The distribution $P(s)$ for a 500 sites scale-free graph with $\lambda=4(m=2)$. A clear transition from Wigner to Poisson is observed as a function of disorder.

An example for such a transition is presented in Fig. 11 where a scale-free graph with $\lambda=4$ and $m=2$ was considered. As $W$ increases $P(s)$ shifts toward the Poisson distribution. Additional hallmark features of the Anderson transition, such as the fact that all curves intersects at $s=2$ and the peak of the distribution "climbs" along the Poisson curve for larger values of $W$ are also apparent. Similar transition from Wigner to Poisson statistics is seen also for the other networks considered in this study.

The transition point can be determined more accurately from calculating [30]

$$
\gamma=\frac{\int_{2}^{\infty} P(s) d s-\int_{2}^{\infty} P_{w}(s) d s}{\int_{2}^{\infty} P_{p}(s) d s-\int_{2}^{\infty} P_{w}(s) d s}
$$

where $\gamma \rightarrow 0$ as the distribution tends toward the Wigner distribution, and $\gamma \rightarrow 1$ if the distribution approaches the Poisson distribution. One expects that as the system size increases, the finite size corrections will become smaller resulting in a distribution closer to a Wigner distribution in the metallic regime and to Poisson in the localized one. At the transition point the distribution should be independent of the system size. In Fig. 2 we plot the behavior of $\gamma$ as function of $W$ for several sizes of a scale free graph. Indeed, $\gamma$ decreases with system size for small values of $W$ while it increases with size for large values of $W$.

All curves should cross around a particular value of disorder signifying the critical disorder. From finite size scaling arguments [30] one expects that $\gamma$ around the critical disorder will depend on the the disorder and network size, $L$, in the following way:

$$
\gamma(W, L)=\gamma\left(W_{c}, L\right)+C\left|\frac{W}{W_{c}}-1\right| L^{1 / \nu},
$$




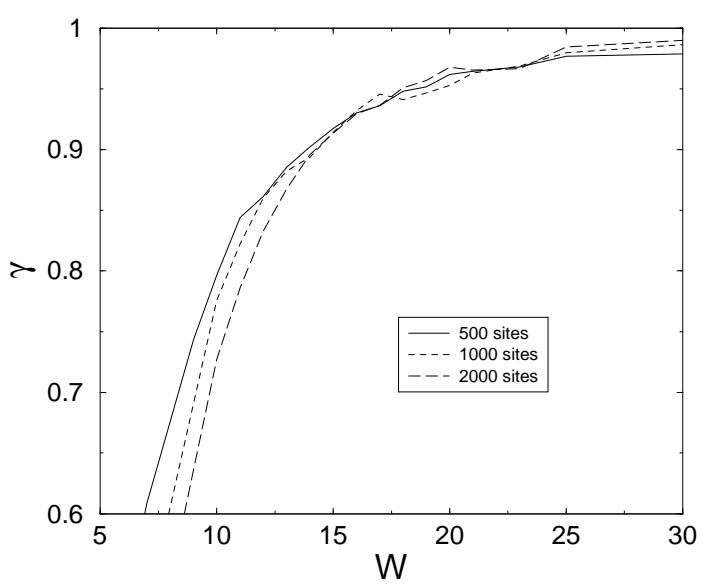

FIG. 2: $\gamma$ as function of $W$ for different SF graphs sizes $(\lambda=4$ and $m=2$ ). The typical behavior for finite size transition is seen, where a crossing in the size dependence of $\gamma$ between the metallic (small values of $W$ ) and localize (large value of $W)$ regime is seen.

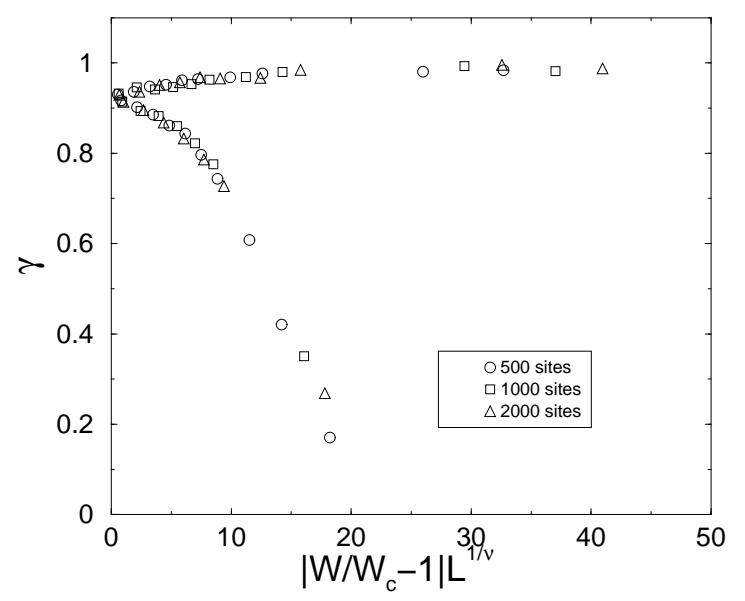

FIG. 3: The scaling of $\gamma$ according to Eq. (7) for different SF, $\lambda=4, m=2$, networks sizes. Two branches, corresponding to the metallic and localized regimes, appear.

where $C$ is a constant. This relation enables us to extract both the critical disorder $W_{c}$ and the critical index $\nu$. Scaling of the numerical data according to Eq. (17) yields two branches corresponding to the metallic and localized regimes, that are clearly seen in Fig. [3] The estimated values of $\nu$ and $W_{c}$ (see Table I) are extracted by fitting the branches to a 4 th order polynomial.

\begin{tabular}{|c|c|c|c|c|}
\hline Network & $\langle k\rangle$ & $l$ & $W_{c}$ & $\nu$ \\
\hline Scale-Free, $\lambda=4, m=2$ & 2.97 & 12.46 & $15.7 \pm 0.9$ & $0.55 \pm 0.11$ \\
\hline Random-Regular (RR) & 3 & 11.8 & $11.9 \pm 0.26$ & $0.66 \pm 0.08$ \\
\hline Erdös-Rényi & 3 & 9.45 & $20.5 \pm 0.23$ & $0.68 \pm 0.08$ \\
\hline Cayley-Tree & 3 & 10 & $11.44 \pm 0.06$ & $0.51 \pm 0.045$ \\
\hline Cayley-Tree with loops & 3.05 & 10 & $12.4 \pm 0.1$ & $0.54 \pm 0.075$ \\
\hline RR "double peak" & 3.1 & 10.28 & $14.1 \pm 0.3$ & $0.85 \pm 0.41$ \\
\hline
\end{tabular}

TABLE I: Networks showing the localization transition. The value of $l$ is for $N=1000$.

\section{RESULTS AND DISCUSSION}

The calculations for all networks mentioned above are performed for $M$ different realizations, where $M=1000,400,200, \ldots, 50$ for the corresponding number of nodes: $N=200,500,1000, \ldots, 4000$. Except for the Cayley-tree networks for which $M=$ $4000,2000,1000, \ldots, 125,64$ for the corresponding tree sizes: $\quad N=63,127,255, \ldots, 2047,4095$ or $L=$ $6,7,8, \ldots, 11,12$ (where $L$ is the number of "generations" of the tree). Another exception is for Erdös-Rényi graphs in which $\langle k\rangle$ is between 3 and 3.5. The low connectivity of the graphs, results in one main cluster and relatively large number of not-connected nodes (about 5\%). Thus, the calculations are made only for the largest cluster of each realization, since a procedure that considers all the nodes is skewed by the eigenvalues of small disconnected clusters 31]. A clear localization transition is observed for a group of graphs which are all characterized by an average degree $\langle k\rangle$ smaller than 3.1, and an averaged last occupied shell $l$ (for $N=1000$ sites) larger or equal to 9.45. The results are summarized in Table I.

The results for all the graphs (including those which show no clear signs of transition) can be scaled according to their average degree $\langle k\rangle$. The higher the value of $\langle k\rangle$ is - the higher is the value of $W$ needed in order to obtain a specific value of $\gamma$. Thus, the higher the average degree, the more metallic the system is, which makes sense. A cross section at $\gamma=0.6$ of all curves is shown in Fig 4 as a function of $\langle k\rangle$. The $\langle k\rangle$ of the networks studied in Fig. 4 as well as the averaged last occupied shell $l$ for $N=1000$ sites are presented in Table II.

The following observations can be gleaned out of the data for the different networks:

(1) For all the networks that show a metal-insulator transition, $\nu$ is of order $1 / 2$ except for the Random-Regular "double-peak" network which is the one with the highest value of connectivity that still shows a clear transition. A critical index of $\nu=0.5$ is expected for a system of infinite dimensionality. At $\langle k\rangle=3.1$ the value of $\nu$ is significantly higher, but so is the estimate of the error bar. On the other hand for the Erdös-Rényi graph with $\langle k\rangle=3.1$ no clear transition is observed.

(2) All networks with connectivity above 3.1 do not show clear signs of a metal-insulator transition. Nevertheless, 


\begin{tabular}{|c|c|c|}
\hline Network & $\langle k\rangle$ & $l$ (for $N=1000)$ \\
\hline \hline Scale-Free $, \lambda=4, m=2$ & 2.97 & 12.46 \\
\hline Random-Regular & 3 & 11.8 \\
\hline $\begin{array}{c}\text { Random-Regular "double peak" } \\
(p=0.95 \rightarrow k=3, p=0.05 \rightarrow k=5)\end{array}$ & 3.1 & 10.28 \\
\hline Cayley-Tree & 3 & 10 \\
\hline Cayley-Tree with loops & 3.05 & 10 \\
\hline Erdös-Rényi & 3 & 9.45 \\
\hline Scale-Free, $\lambda=3.5, m=2$ & 3.28 & 9.36 \\
\hline Erdös-Rényi & 3.1 & 9.31 \\
\hline Erdös-Rényi & 3.2 & 9.03 \\
\hline $\begin{array}{c}\text { Random-Regular "double peak" } \\
(p=0.8 \rightarrow k=3, p=0.2 \rightarrow k=5)\end{array}$ & 3.4 & 9 \\
\hline Erdös-Rényi & 3.5 & 8.33 \\
\hline Random-Regular "double peak" & & \\
$(p=0.95 \rightarrow k=3, p=0.05 \rightarrow k=10)$ & 3.35 & 7.99 \\
\hline Erdös-Rényi & 4 & 7.51 \\
\hline Scale-Free, $\lambda=5, m=3$ & 4 & 7.37 \\
\hline Scale-Free $, \lambda=4, m=3$ & 4.5 & 6.05 \\
\hline $\begin{array}{c}\text { Random-Regular "double peak" } \\
(p=0.8 \rightarrow k=3, p=0.2 \rightarrow k=10)\end{array}$ & 4.4 & 6.13 \\
\hline Erdös-Rényi & 5 & 6.31 \\
\hline Erdös-Rényi & 7.5 & 5.02 \\
\hline Erdös-Rényi & 10 & 4.1 \\
\hline
\end{tabular}

TABLE II: The average connectivity $\langle k\rangle$ of all the networks considered in this study, as well as the averaged last occupied shell $l$ for $N=1000$ sites.

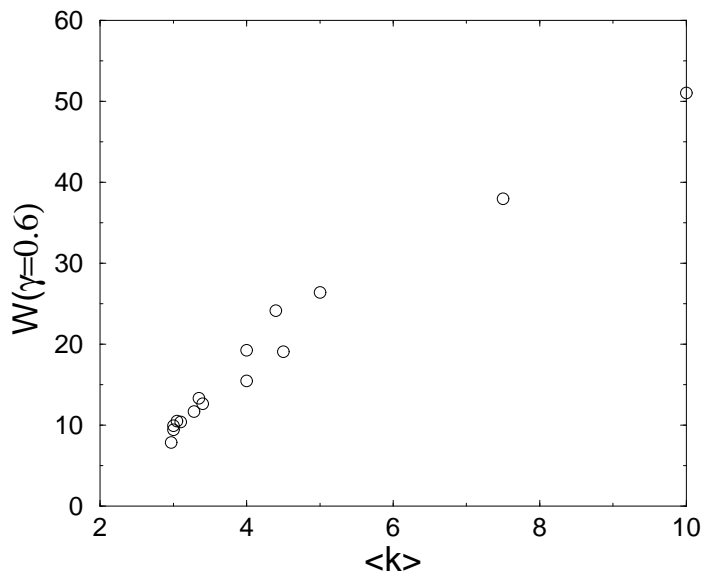

FIG. 4: The $\gamma$ curves of the checked networks can be ordered by their $\langle k\rangle$. The values of their $W(\gamma=0.6)$ are presented as a function of $\langle k\rangle$. One can notice the increasing $W$ with $\langle k\rangle$. one should be rather careful in interpreting this observation since, as is clear from Table II, larger values of $\langle k\rangle$ lead to smaller size, $l$, of the network for the same number of nodes. Moreover, from the two networks which have the same $\langle k\rangle=3.1$, only the one with the higher value of $l$ shows clear signs of the metal insulator transition. Thus, the absence of transition may be an artifact of the small size of networks with high average connectivity. (3) The critical disorder $W_{c}$ fluctuates in the range of $12-20$ (Table I). Due to the small range of $\langle k\rangle(2.97-3.1)$, it is hard to determine any relation between $k$ and $W_{c}$.

(4) On the other hand, there is a clear relation between the amount of disorder needed in order to reach a particular value of $\gamma$ (i.e., the value of $W$ needed to reach a certain degree of localization) and $\langle k\rangle$. As can be seen in Fig. 4 a linear dependence $W(\gamma=0.6) \propto\langle k\rangle$ is observed.

Thus, the gross features of the Anderson metalinsulator transition are similar for a wide range of different networks. The critical index for all the networks studied here are within the range expected for a system of infinite dimensionality, and the connectivity influences the degree of localization. Thus, complex networks are an example of a topology for which the critical index follows the mean field prediction, but the critical disorder can be tuned by the connectivity. On the other hand, the fact that networks with high connectivity are very compact raises the problem of identifying the transition point. It is hard to extend the usual finite-size scaling method to networks with high connectivity since the number of sites grows very rapidly with size, while for small network sizes the crossover behavior of the $\gamma$ curves is very noisy. This results in an inability to clearly identify the Anderson transition, although it can not be ruled out the possibility that there is a critical connectivity for complex networks above which no transition exist.

We acknowledge very useful discussions with J. W. Kantelhardt and helpful correspondence with Y.V. Fyodorov, as well as support from the Israel Academy of Science (Grant 877/04), the ONR, and the Israeli Center for Complexity Science. This work was also supported by the European research NEST Project No. DYSONET 012911.

[1] P. W. Anderson, Phys. Rev. 109, 1492 (1958); D.J. Thouless, Phys. Rep. 13, 93 (1974).

[2] see these reviews and references within: G. Bergmann, 
Phys. Rep. 107, 1 (1984); P. A. Lee and T. V. Ramakrishnan, Rev. Mod. Phys. 57, 287 (1985); R. Berkovits and S. Feng, Phys. Rep. 238, 135 (1994); M. C. W. van Rossum and T. M. Nieuwenhuizen, Rev. Mod. Phys. 71, 313 (1999).

[3] E. Abrahams, P. W. Anderson, D. C. Licciardello, and T. V. Ramakrishnan, Phys. Rev. Lett. 42, 673 (1979).

[4] C. Castellani, C. DiCastro, and L. Peliti, J. Phys. A 19, L1099 (1986); H. Kunz and B. Souillard, J. Phys. Lett. 44, L503 (1983); J. P. Straley, Phys. Rev. B 28, 5393 (1983); T. Lukes, J. Phys. C 12, L797 (1979); K. B. Efetov, Sov. Phys. JETP 61, 606 (1985).

[5] A. D. Mirlin and Y. V. Fyodorov, Phys. Rev. Lett. 72, 526 (1994).

[6] B. Kramer and A. MacKinnon, Rep. Prog. Phys. 56, 1496 (1993).

[7] K. Slevin, T. Ohtsuki and T. Kawarabayashi, Phys. Rev. Lett. 84, 3915 (2000).

[8] I. K. Zharekeshev and B. Kramer, Ann. Phys. (Leipzig) 7, 442 (1998).

[9] M. Schreiber and H. Grussbach, Phys. Rev. Lett. 76, 1687 (1996).

[10] R. Albert, H. Jeong, and A.L. Barabási, Nature, 406, 6794 (2000).

[11] For a recent review see: R. Albert and A.L. Barabási, Rev. Mod. Phys. 74, 47 (2002).

[12] S. N. Dorogovtsev and J. F. F. Mendes, Evolution of Networks - From Biological Nets to the Internet and $W W W$ (Oxford University Press, 2003)

[13] R. Pastor-Satorras and A. Vespignani, Evolution and Structure of the Internet : A Statistical Physics Approach (Cambridge University Press, 2004)

[14] R. Cohen, K. Erez, D. ben Avraham, and S. Havlin, Phys. Rev. Lett. 85, 4626 (2000); Phys. Rev. Lett. 86, 3682 (2001).

[15] R. Cohen and S. Havlin, Phys. Rev. Lett. 90, 058701 (2003).
[16] B. Bollobas and O. Riordan in Handboook of graphs and networks, eds. S. Bornholdt and H.G. Schuster (WileyVCH, Berlin,2002)

[17] M. Molloy and B. Reed, Combinatorics, Probability and Computing 7, 295 (1998).

[18] D.S. Callaway, M.E.J. Newman, S.H, Strogatz, and D.J. Watts, Phys. Rev. Lett. 85, 5468 (2000).

[19] M.E.J. Newman, S.H, Strogatz, and D.J. Watts, Phys. Rev. E 64, 026118 (2001).

[20] C. P. Zhu and S.-J. Xiong, Phys. Rev. B 62, 14780 (2000).

[21] O. Giraud, B. Georgeot and D. L. Shepelyansky, quant-ph/0503188

[22] M. Sade and R. Berkovits, Phys. Rev. B 68, 193102 (2003).

[23] Y.V. Fyodorov and A.D. Mirlin, Phys. Rev. Lett. 672049 (1991); S.N. Evangelou and E.N. Economou, Phys. Rev. Lett. 68, 361 (1992); Y.V. Fyodorov et. al., J. Phys. I (France) 2, 1571 (1992); Y.V. Fyodorov and A.D. Mirlin, Phys. Rev. B 55, R16001 (1997).

[24] L. A. Braunstein, S. V. Buldyrev, R. Cohen, S. Havlin, and H. E. Stanley, Phys. Rev. Lett. 91, 168701 (2003).

[25] E. Lopez, S. V. Buldyrev, S. Havlin, and H. E. Stanley, Phys. Rev. Lett. 94, 248701 (2005).

[26] E. M. Bollt and D. ben-Avraham, cond-mat/0409465

[27] B. Shapiro, Phys. Rev. Lett. 48, 823 (1982).

[28] P. Erdös and A. Rényi, Publ. Math. Debrecen 6, 290 (1959).

[29] T. Kalisky, R. Cohen, D. ben Avraham, and S. Havlin, in Lecture Notes in Physics: Proceedings of the 23rd LANL-CNLS Conference, "Complex Networks", Santa$\mathrm{Fe}$, 2003, eds. E. Ben-Naim, H. Frauenfelder, and Z. Toroczkai (Springer, Berlin, 2004).

[30] B.I. Shklovskii, B. Shapiro, B.R. Sears, P. Lambrianides, and H.B. Shore, Phys. Rev. B 47, 11487 (1993).

[31] R. Berkovits and Y. Avishai, Phys. Rev. B 53, 16125 (1996). 